\title{
Adverse effect of mild temperature hyperthermia combined with hexamethylenetetramine compared to its effect combined with tirapazamine in the treatment of solid tumors
}

\author{
SHIN-ICHIRO MASUNAGA ${ }^{1}$, KEIZO TANO ${ }^{2}$, JUN NAKAMURA ${ }^{3}$, MASAMI WATANABE $^{2}$, \\ GENRO KASHINO $^{1}$, MINORU SUZUKI ${ }^{1}$, YUKO KINASHI ${ }^{4}$ and KOJI ONO ${ }^{1}$ \\ ${ }^{1}$ Particle Radiation Oncology Research Center, ${ }^{2}$ Particle Radiation Biology, and ${ }^{4}$ Radiation Safety and Control, \\ Research Reactor Institute, Kyoto University, Kumatori, Osaka 590-0494, Japan; ${ }^{3}$ Department of Environmental \\ Sciences and Engineering, University of North Carolina at Chapel Hill, Chapel Hill, NC 27599, USA
}

Received July 1, 2009; Accepted August 20, 2009

DOI: 10.3892/etm_00000027

\begin{abstract}
This study aimed to assess the effect on solid tumors of mild temperature hyperthermia (MTH) combined with hexamethylenetetramine (HMTA) or tirapazamine (TPZ). Squamous cell carcinoma (SCC VII) tumor-bearing mice were continuously administered 5-bromo-2'-deoxyuridine (BrdU) to label intratumor proliferating $(\mathrm{P})$ cells. Mice received HMTA or TPZ through intraperitoneal single or subcutaneous continuous administration, with or without $\mathrm{MTH}\left(40^{\circ} \mathrm{C}, 60 \mathrm{~min}\right)$, followed or not by $\gamma$-ray irradiation or cisplatin treatment. After HMTA or TPZ administration without $\gamma$-ray irradiation or cisplatin treatment, immediately after $\gamma$-ray irradiation, or $1 \mathrm{~h}$ after cisplatin treatment, the response of quiescent (Q) cells was assessed in terms of micronucleus frequency using immunofluorescence staining for BrdU. The response of the total $(\mathrm{P}+\mathrm{Q})$ tumor cells was determined based on a comparison with non-BrdU-treated tumors. Without MTH, HMTA and TPZ had a nearly equal radiosensitizing and cisplatin sensitivity-enhancing effect on both total and Q cells. With MTH, radio- and cisplatin-sensitizing effects by HMTA were reduced, particularly in the Q cells. In contrast, the enhancing effects of TPZ were increased, particularly in the Q cells. Continuous administration of HMTA and TPZ resulted in higher radio- and cisplatin-sensitizing effects than intraperitoneal single administration. In terms of tumor cytotoxicity as a whole, including $\mathrm{Q}$ cells, the administration of $\gamma$-ray irradiation or cisplatin treatment combined with continuous HMTA administration is promising, taking into account the clinical use of HMTA. However, MTH should not be combined with HMTA administration.
\end{abstract}

Correspondence to: Dr Shin-Ichiro Masunaga, Particle Radiation Oncology Research Center, Research Reactor Institute, Kyoto University, 2-1010, Asashiro-nishi, Kumatori-cho, Sennan-gun, Osaka 590-0494, Japan

E-mail: smasuna@rri.kyoto-u.ac.jp

Key words: mild temperature hyperthermia, hexamethylenetetramine, tirapazamine, quiescent cell, hypoxia

\section{Introduction}

Hyperthermia is a heat treatment that directly targets cancer cells or the environment surrounding them (1). According to the tenets of hyperthermic oncology, significant tumor cell killing could theoretically be achieved if cells or tissues were heated to over $42^{\circ} \mathrm{C}$ for $1 \mathrm{~h}$ or more (2). It is speculated that such heat treatment would induce radio- and chemosensitization, in part by inhibiting DNA damage repair (1). However, clinical experience over the past 25 years has shown that it is not possible to routinely achieve thermal doses of over $42^{\circ} \mathrm{C}$ for $1 \mathrm{~h}$ or more. It is now known that, during typical hyperthermia treatments with currently available heating technologies (except for thermal ablation), cytotoxic temperatures are only achieved in small sub-volumes of tumors (3). Meanwhile, until recently the effects of mild temperature hyperthermia (MTH) $\left(39-42^{\circ} \mathrm{C}\right.$ for 1-2 h) on tissue have largely been ignored. However, MTH has subtle effects, including heat-mediated tumor reoxygenation (4) and the inhibition of sublethal and potentially lethal damage repair (5), which provide a very strong rationale for using MTH in combination with radiotherapy. In addition, the physiological and cellular effects of MTH can improve the delivery of drug vehicles (6), activate promoters for heat-mediated gene therapy (7) and increase immune response to tumors through a variety of mechanisms (8). Indeed, MTH has been reported to increase the response of tumors, in particular of quiescent (Q)-cell populations, to radiation or chemotherapeutic agents, in part by improving oxygenation through an increase in tumor blood flow (9).

Tumor hypoxia results from limited oxygen diffusion (chronic hypoxia) or limited perfusion (acute hypoxia, transient hypoxia or ischemic hypoxia) (10). Chronically hypoxic tumor cells existing at the rim of the oxygen diffusion distance are killed by just a single administration of tirapazamine (TPZ), a lead compound identified in the development of a bioreductive hypoxic cytotoxin (Fig. 1), while acutely hypoxic tumor cells occurring sporadically throughout solid tumors are killed by long-term continuous administration of TPZ. Therefore, the long-term continuous administration of TPZ is capable of killing both chronically and acutely hypoxic tumor cells (9). 
Although TPZ has enhanced some standard clinical regimens, the results are variable and, with certain combinations, toxicity is enhanced (11). Neutropenia is commonly reported, but usually within a tolerable range. The most frequent non-hematological toxicities reported are nausea, vomiting, diarrhea and skin rash. Two additional unusual toxicities are reversible deafness and muscle cramping (11). According to a recently reported in vivo animal study, the addition of TPZ to cisplatin/radiotherapy treatment caused a significant increase in toxicity, in particular to the heart, liver, kidney and stomach, and had only moderate effects on the tumor (12). As a result, TPZ has exhibited no clear beneficial effects compared to conventional drugs in clinical trials conducted to date.

Formaldehyde preserves or fixes tissues or cells by irreversibly crosslinking primary amine groups in proteins with other nearby nitrogen atoms in protein or DNA through a -CH2- linkage (13). Hexamethylenetetramine (HMTA) (Fig. 1), an acid-dependent formaldehyde donor, has been used as an antiseptic for urinary tract infections and is characterized as non-carcinogenic in animals (13). Under hypoxic conditions in solid tumors, pyruvate generated by glycolysis creates a low $\mathrm{pH}$ environment that produces formaldehyde through the dissociation of HMTA (14).

Excepting surgical resection, chemotherapy using cis-diamminedichloroplatinum (cisplatin) and radiotherapy are the most frequently employed anticancer therapeutic modalities applied in the clinical setting. Based on clinical experience and using a previously developed method for selectively detecting the response of $\mathrm{Q}$ cell populations within solid tumors (15), the combined effect of MTH with $\gamma$-ray irradiation or cisplatin treatment in combination with or without HMTA or TPZ administration was evaluated. In addition, the efficacy of continuous administration of HMTA or TPZ versus single injection was examined.

\section{Materials and methods}

Mice and tumors. SCC VII squamous cell carcinomas (Department of Radiotherapy, Kyoto University) derived from $\mathrm{C} 3 \mathrm{H} / \mathrm{He}$ mice were maintained in vitro in Eagle's minimum essential medium supplemented with $12.5 \%$ fetal bovine serum. The cells $\left(1.0 \times 10^{5}\right)$ were inoculated subcutaneously into the left hind legs of 8- to 11-week-old syngeneic female $\mathrm{C} 3 \mathrm{H} / \mathrm{He}$ mice (Japan Animal Co., Ltd., Osaka, Japan). After 14 days, tumors with a diameter of $\sim 1 \mathrm{~cm}$ were selected for use in the experiments. The body weight of the tumor-bearing mice was $22.1 \pm 2.3 \mathrm{~g}$ (mean $\pm \mathrm{SD}$ ). Mice were handled according to the Recommendations for Handling of Laboratory Animals for Biomedical Research, compiled by the Committee on Safety and Ethical Handling Regulations for Laboratory Animal Experiments of Kyoto University. The p53 of the SCC VII tumor cells was wild-type (15).

Labeling with 5-bromo-2'-deoxyuridine. Nine days after inoculation, mini-osmotic pumps (Durect Corporation, Cupertino, CA, USA) containing 5-bromo-2'-deoxyuridine (BrdU) dissolved in physiological saline $(250 \mathrm{mg} / \mathrm{ml})$ were implanted subcutaneously for 5 days to label all proliferating (P)-cells. The percentage of labeled cells in the SCC VII tumors after continuous labeling with BrdU reached a plateau

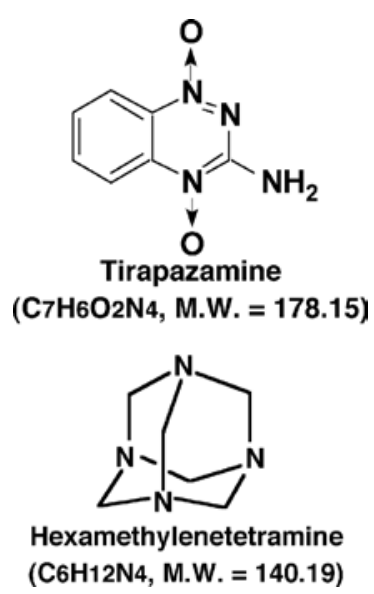

Figure 1. Chemical structure of hexamethylenetetramine (HMTA) and tirapazamine (TPZ).

at $55.3 \pm 4.5 \%$. Intratumor cells not incorporating BrdU after continuous labeling were determined to be Q cells.

Treatment. After labeling with BrdU, TPZ and HMTA dissolved in physiological saline were administered at doses of $0.224 \mathrm{mmol} / \mathrm{kg}$ or $28.5 \mathrm{mmol} / \mathrm{kg}$, respectively, by single intraperitoneal injection or by continuous administration for $24 \mathrm{~h}$ by means of subcutaneously implanted mini-osmotic pumps. MTH $\left(40^{\circ} \mathrm{C}, 60 \mathrm{~min}\right)$ was initiated immediately after single intraperitoneal injection or was performed during the continuous administration, immediately before $\gamma$-ray irradiation or cisplatin treatment. Not all tumors received MTH prior to $\gamma$-ray irradiation or cisplatin treatment.

To detect the radiosensitizing effects of TPZ or HMTA, whole body irradiation with $\gamma$-rays was performed at a dose of 4 or 20 Gy using a cobalt- $60 \gamma$-ray irradiator at a dose rate of $\sim 2.5 \mathrm{~Gy} / \mathrm{min}$. Cisplatin was intraperitoneally administered at a dose of $0.5 \mathrm{LD}_{50}$ (the dose required to kill $50 \%$ of cells, $\mathrm{LD}_{50}=17.7 \mathrm{mg} / \mathrm{kg}$ ) to detect the cisplatin sensitivity-enhancing effects of TPZ or HMTA. Cytotoxicity was assessed in terms of cell survival and induced micronucleus (MN) frequency following the intraperitoneal or continuous administration of TPZ or HMTA alone at the above doses (16).

For MTH, the tumors grown in the left hind legs of mice were heated at $40^{\circ} \mathrm{C}$ for $60 \mathrm{~min}$ by immersion of the tumorbearing foot in a water bath as follows: the mouse was held in a specially constructed device with the tail and right leg firmly fixed with adhesive tape. The left tumor-bearing leg was pulled down by a special sinker ( $45 \mathrm{~g})$ affixed to the skin of the toe with Superglue (Arone-arufa, Konishi Co., Osaka, Japan). The mouse was placed in a circulating water bath maintained at the desired temperature, and was air-cooled during the heat treatment (17). Temperatures at the tumor center equilibrated within 3-4 min of immersion in the water bath and remained $0.2-0.3^{\circ} \mathrm{C}$ below bath temperature. The water bath temperature was therefore maintained at $0.3^{\circ} \mathrm{C}$ above the desired tumor temperature.

In mice receiving HMTA or TPZ treatment alone, the implanted tumors were excised $1 \mathrm{~h}$ after the single intraperitoneal injection or after the 24-h continuous subcutaneous infusion. In mice receiving the combination of each drug with $\gamma$-ray irradiation, implanted tumors were excised immediately 
after irradiation. For the combination with cisplatin treatment, implanted tumors were excised $1 \mathrm{~h}$ after the administration of cisplatin.

The above-mentioned sequences and the timing of each treatment were based on data obtained from preliminary experiments. Procedures were appropriate and were carried out successfully $(9,16)$.

Immunofluorescence staining of BrdU-labeled cells and micronucleus assay. Tumors excised from the mice administered $\mathrm{BrdU}$ were minced and trypsinized with $0.05 \%$ trypsin and $0.02 \%$ ethylenediamine-tetraacetic acid (EDTA) in phosphate-buffered saline (PBS) at $37^{\circ} \mathrm{C}$ for $15 \mathrm{~min}$. Tumor cell suspensions were incubated for $72 \mathrm{~h}$ in tissue culture dishes containing complete medium and $1.0 \mu \mathrm{g} / \mathrm{ml}$ of cytochalasin-B in order to inhibit cytokinesis while allowing nuclear division. The cultures were subsequently trypsinized and the cell suspensions were fixed. After the centrifugation of the fixed cell suspensions, the cell pellet was resuspended with cold Carnoy's fixative (ethanol:acetic acid, 3:1 volume). The suspension was then placed on a glass microscope slide and the sample was dried at room temperature. The slides were treated with $2 \mathrm{M}$ hydrochloric acid for $60 \mathrm{~min}$ at room temperature to dissociate the histones and to partially denature the DNA, and were then immersed in borax-borate buffer $(\mathrm{pH} \mathrm{8.5)}$ to neutralize the acid. BrdU-labeled tumor cells were detected by indirect immunofluorescence staining using monoclonal anti-BrdU antibody (Becton Dickinson, San Jose, CA, USA) and fluorescein isothiocyanate (FITC)-conjugated antimouse IgG antibody (Sigma, St. Louis, MO, USA). To observe the double staining of tumor cells with green-emitting FITC and red-emitting propidium iodide (PI), cells on the slides were treated with PI $(2 \mu \mathrm{g} / \mathrm{ml}$ in PBS) and monitored under a fluorescence microscope.

Since cytochalasin-B inhibits cytokinesis while allowing nuclear division, cultured tumor cells were transformed into binuclear cells after the first cell division. When cell division is disrupted or chromosomes have been broken or damaged by chemicals or radiation, the distribution of genetic material between the two-daughter nuclei during cell division is affected, and pieces or entire chromosomes fail to be included in either of the two-daughter nuclei. Genetic material that is not incorporated into a new nucleus forms its own 'micronucleus'. Micronucleus (MN) frequency in cells not labeled with BrdU was examined by counting the micronuclei in the binuclear cells that showed only red fluorescence. The MN frequency was defined as the ratio of the number of micronuclei in the binuclear cells to the total number of binuclear cells observed (15). MN frequency may also be used to detect sensitivity to chemotherapeutic agents (15).

The ratios obtained in tumors not pre-treated with BrdU revealed the $\mathrm{MN}$ frequency at all phases in the total $(\mathrm{P}+\mathrm{Q})$ tumor cell population. More than $400(444 \pm 31)$ binuclear cells were counted to determine $\mathrm{MN}$ frequency. The MN frequencies in control cells receiving absolutely no treatment were $0.056 \pm 0.005$ and $0.081 \pm 0.011$, with respect to the total tumor and Q cell populations in the SCC VII tumors. For baseline correction, the induced $\mathrm{MN}$ frequency (the $\mathrm{MN}$ frequency of the treated tumors minus that of the non-treated control tumors) was used to exclude the MN frequency in control cells receiving absolutely no treatment.

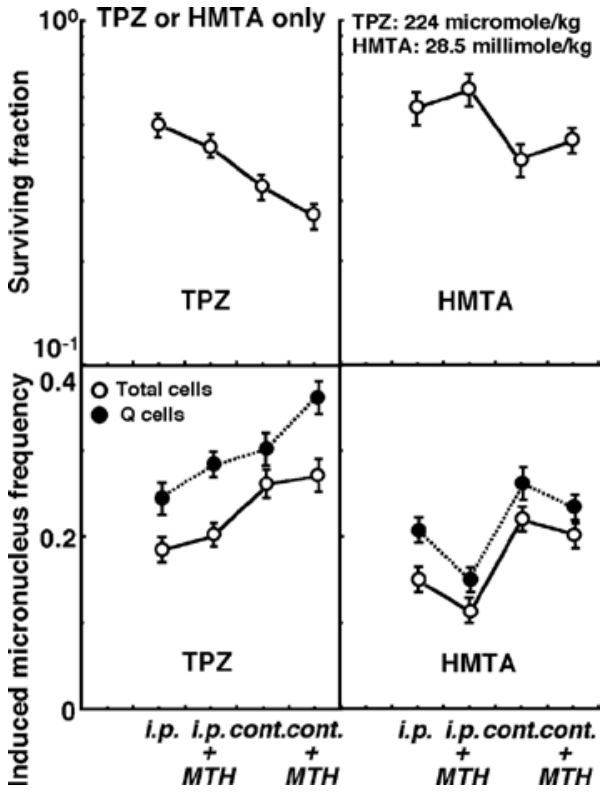

Figure 2. Changes in the clonogenic cell surviving fraction of the total tumor cell population (upper panels) and the induced micronucleus frequencies of the total and quiescent (Q)-cell populations (lower panels) following intraperitoneal (i.p.) single or subcutaneous 24-h continuous (cont.) administration of tirapazamine (TPZ) (left panels) or hexamethylenetetramine (HMTA) (right panels), in combination with or without mild temperature hyperthermia (MTH) $\left(40^{\circ} \mathrm{C}, 60 \mathrm{~min}\right)$. Assays were performed $1 \mathrm{~h}$ after i.p. single administration or after the 24-h cont. administration. Bars represent the SD.

Clonogenic cell survival assay. The clonogenic cell survival assay was also performed in the mice not pre-treated with BrdU using an in vivo-in vitro assay method. Tumors were excised from the mice, minced and disaggregated by stirring for $20 \mathrm{~min}$ at $37^{\circ} \mathrm{C}$ in PBS containing $0.05 \%$ trypsin and $0.02 \%$ EDTA. Through these procedures, single tumor cell suspensions were obtained from the whole tumor. The cell yield was $(4.5 \pm 1.1) \times 10^{7} / \mathrm{g}$ tumor weight. Appropriate numbers of viable tumor cells from the single cell suspension were plated on 60 or $100-\mathrm{mm}$ tissue culture dishes. After 12 days, the colonies were fixed with ethanol, stained with Giemsa and counted. Plating efficiency under the no treatment control condition was $52.0 \pm 4.5 \%$.

The sensitivity of Q cells was assessed in terms of the MN frequency using immunofluorescence staining for BrdU, while the sensitivity of the total $(\mathrm{P}+\mathrm{Q})$ tumor cells was determined based on a comparison with the non-BrdU-treated tumors based on MN frequency and clonogenic cell survival.

Four mice were used to assess each set of conditions, and each experiment was repeated at least twice. To examine the differences between pairs of values, the Student's t-test was used when variances between the two groups were assumed to be equal; otherwise, Welch's t-test was used. P-values were determined by two-sided tests.

\section{Results}

Changes in the clonogenic cell surviving fraction of the total tumor cell population (upper panels), as well as changes in the induced $\mathrm{MN}$ frequencies of the total and Q cell populations (lower panels) following intraperitoneal single or subcutaneous 


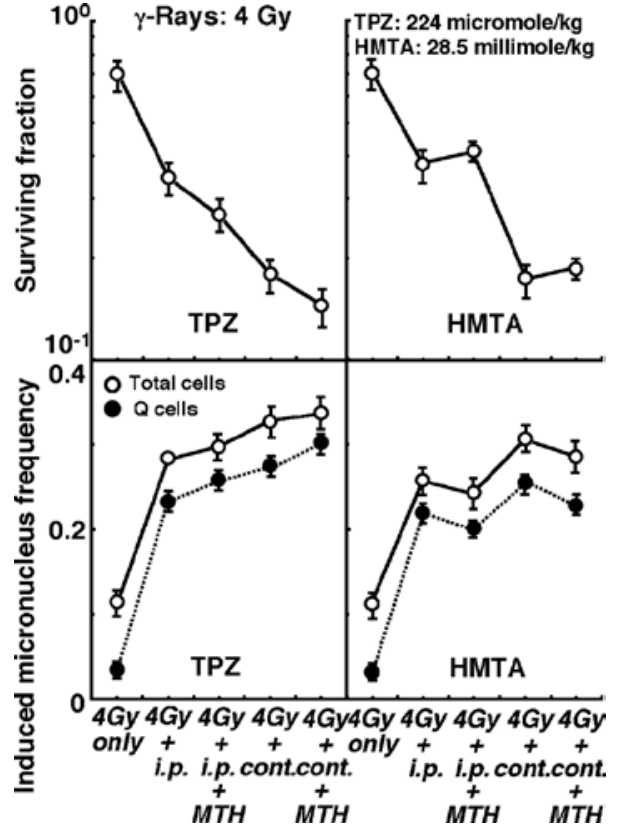

Figure 3. Changes in the clonogenic cell surviving fraction of the total tumor cell population (upper panels) and the induced micronucleus frequencies of the total or quiescent (Q)-cell populations (lower panels) immediately after $\gamma$-ray irradiation at a dose of $4 \mathrm{~Gy}$, in combination with or without pre-irradiation intraperitoneal (i.p.) single or subcutaneous continuous (cont.) administration of tirapazamine (TPZ) $(0.224 \mathrm{mmol} / \mathrm{kg}$; left panels) or hexamethylenetetramine (HMTA) $(28.5 \mathrm{mmol} / \mathrm{kg}$; right panels) in further combination with or without mild temperature hyperthermia $(\mathrm{MTH})\left(40^{\circ} \mathrm{C}, 60 \mathrm{~min}\right)$. Assays were performed immediately after irradiation, $1 \mathrm{~h}$ after i.p. single administration or after the 24-h cont. administration. Bars represent the SD.

24-h continuous administration of TPZ (left panels) or HMTA (right panels) in combination with or without $\mathrm{MTH}\left(40^{\circ} \mathrm{C}\right.$, 60 min) are shown in Fig. 2.

Under all conditions, the sensitivity of the $\mathrm{Q}$ cells was significantly higher than that of the total cells, regardless of whether TPZ or HMTA was used $(\mathrm{P}<0.05)$. Concerning both TPZ and HMTA, sensitivity was significantly higher with continuous administration than with intraperitoneal single administration $(\mathrm{P}<0.05)$. Sensitivity to TPZ was enhanced by the combination with MTH, whether TPZ was administered by single or continuous administration. In contrast, sensitivity to HMTA was decreased in combination with MTH.

Changes in the clonogenic cell surviving fraction of the total tumor cell population (upper panels) and the induced MN frequencies of the total or Q cell populations (lower panels) immediately after $\gamma$-ray irradiation in combination with or without pre-irradiation intraperitoneal single or subcutaneous continuous administration of TPZ (left panels) or HMTA (right panels) in further combination with or without MTH, are shown in Figs. 3 (4 Gy irradiation dose) and 4 (20 Gy irradiation dose).

Concerning both TPZ and HMTA, subcutaneous continuous administration compared with intraperitoneal single administration induced significantly higher sensitivity to $\gamma$-rays at doses of both 4 and $20 \mathrm{~Gy}$ in the total and the Q cells $(\mathrm{P}<0.05)$. Regardless of whether TPZ was administered by single or continuous administration, with $\gamma$-ray irradiation at doses of 4 and $20 \mathrm{~Gy}$, the combination with MTH enhanced

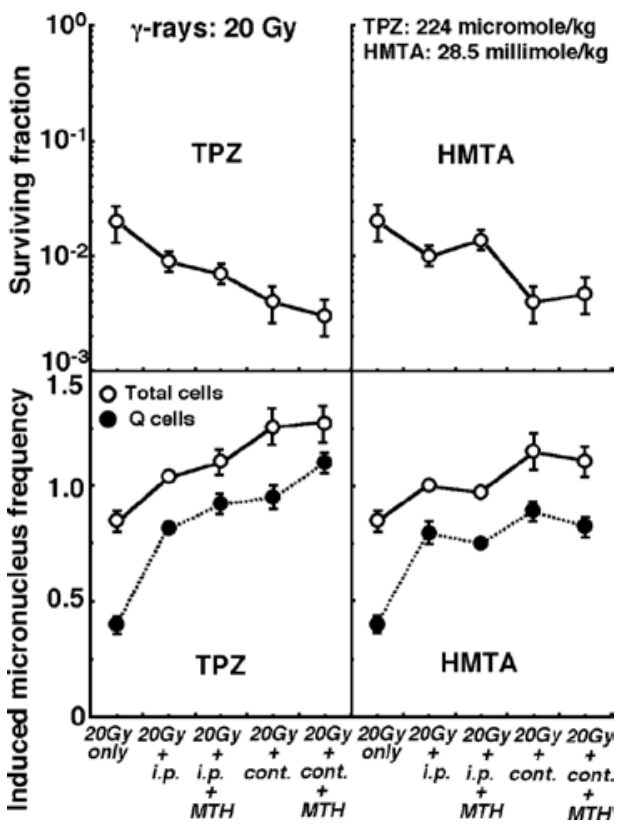

Figure 4. Changes in the clonogenic cell surviving fraction of the total tumor cell population (upper panels) and the induced micronucleus frequencies of the total or quiescent (Q)-cell populations (lower panels) immediately after $\gamma$-ray irradiation at a dose of $20 \mathrm{~Gy}$, in combination with or without pre-irradiation intraperitoneal (i.p.) single or subcutaneous continuous (cont.) administration of tirapazamine (TPZ) $(0.224 \mathrm{mmol} / \mathrm{kg}$; left panels) or hexamethylenetetramine (HMTA) $(28.5 \mathrm{mmol} / \mathrm{kg}$; right panels) in further combination with or without mild temperature hyperthermia $(\mathrm{MTH})\left(40^{\circ} \mathrm{C}\right.$, $60 \mathrm{~min}$ ). Assays were performed immediately after irradiation. Bars represent the SD.

$\gamma$-ray sensitivity, especially in the Q cells. In contrast, $\gamma$-ray sensitivity was reduced by HMTA in combination with MTH, especially in the Q cells.

Changes in the clonogenic cell surviving fraction of the total tumor cell populations (upper panels) and the induced MN frequencies of the total or Q cell populations (lower panels) $1 \mathrm{~h}$ after intraperitoneal single administration of cisplatin in combination with or without pre-treatment intraperitoneal single or subcutaneous continuous administration of TPZ (left panels) or HMTA (right panels) in further combination with or without MTH are shown in Fig. 5.

Regardless of whether TPZ or HMTA was used, subcutaneous continuous administration induced significantly higher sensitivity to cisplatin in both the total and Q cells compared to intraperitoneal single administration $(\mathrm{P}<0.05)$. Concerning both single or continuous administration of TPZ, the combination with MTH enhanced cisplatin sensitivity, especially in the $\mathrm{Q}$ cells. In contrast, cisplatin sensitivity in combination with HMTA was reduced in further combination with MTH, especially in the Q cells.

Without MTH, the enhancing effects of continuous administration compared with intraperitoneal single administration were more marked in total cells than in Q cells, regardless of whether HMTA or TPZ was administered in combination with $\gamma$-ray irradiation or cisplatin treatment. In cells treated with TPZ or HMTA alone, both drugs had nearly the same cytotoxic effect in terms of cell survival and induced MN frequency when administered by intraperitoneal single administration. 


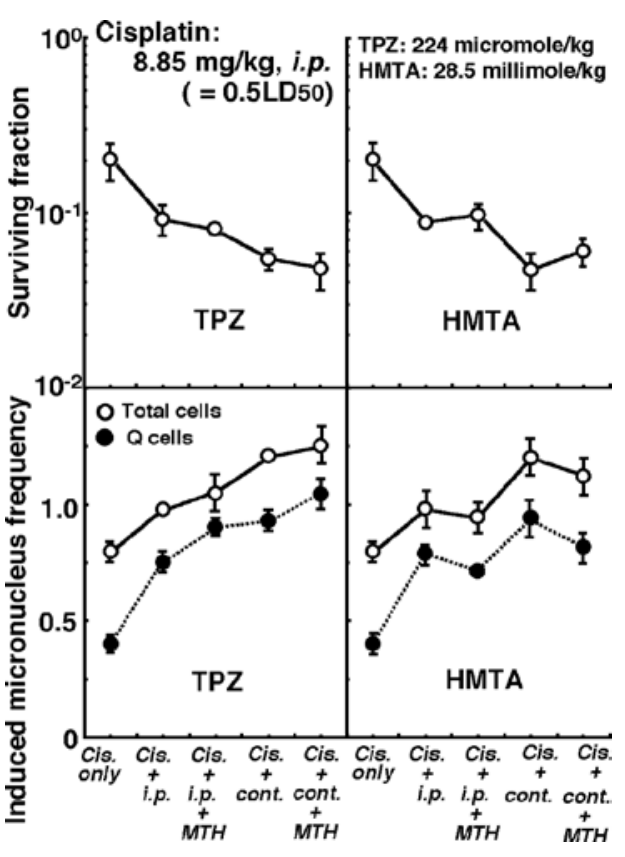

Figure 5. Changes in the clonogenic cell surviving fraction of the total tumor cell populations (upper panels) and the induced micronucleus frequencies of the total or quiescent (Q)-cell populations (lower panels) $1 \mathrm{~h}$ after intraperitoneal (i.p.) single administration of cisplatin (Cis.) $(8.85 \mathrm{mg} / \mathrm{kg})$ in combination with or without pre-treatment i.p. single or subcutaneous continuous (cont.) administration of tirapazamine (TPZ) $(0.224 \mathrm{mmol} / \mathrm{kg}$; left panels) or hexamethylenetetramine (HMTA) $(28.5 \mathrm{mmol} / \mathrm{kg}$; right panels) in further combination with or without mild temperature hyperthermia (MTH) $\left(40^{\circ} \mathrm{C}, 60 \mathrm{~min}\right)$. Assays were performed $1 \mathrm{~h}$ after cisplatin treatment. Bars represent the $\mathrm{SD}$

\section{Discussion}

Formaldehyde is a naturally occurring biological compound present in all tissues, cells and bodily fluids (13). It functions as a key intermediate in the 'one-carbon pool' used for the biosynthesis of purines, thymidine and some amino acids. It is usually rapidly metabolized by reduction, oxidation and reduced glutathione-dependent pathways (13). However, saturation in formaldehyde metabolism leads to DNA damage. It has been shown that cells exposed to formaldehyde exhibit, as a major form of DNA damage, DNA-protein crosslinks (18). In a limited oxygen environment, pyruvate generated by glycolysis in the cell cytoplasm is preferentially converted into lactic acid by lactate dehydrogenase, which creates a low $\mathrm{pH}$ environment ( $\mathrm{pH}$ 6.4-6.8) (19). Furthermore, through the Warburg effect, many cancer cells vigorously consume glucose and preferentially produce lactic acid, even in the presence of adequate oxygen (19). Thus, due to the extracellular acidic conditions adjacent to solid tumors, an acid-dependent formaldehyde donor, HMTA, dissociates to release formaldehyde into nearby tissues.

HMTA has been employed as an antiseptic for the treatment of urinary tract infections, and has been studied in patients with maxillofacial phelegmons and for use as a prophylactic agent against recurrent acute cystitis $(13,14)$. HMTA has been demonstrated to be well tolerated, even at doses of up to $5 \mathrm{~g} / \mathrm{kg} /$ day $(14,20)$. Since HMTA is known to hydrolyze under cellular conditions and to release 6 molecules of formaldehyde in a pHdependent manner (14), we examined HMTA for its potential as a formaldehyde-releasing prodrug for damaging DNA within a tumor cell. As shown in our previous report, $\gamma$-ray irradiation and cisplatin treatment combined with continuous HMTA or TPZ administration is promising in terms of the tumor cellkilling effect as a whole (including Q cells) (16).

The $\mathrm{MN}$ frequencies of cells from tumors treated with MTH only showed that MTH was not capable of inducing direct thermal cytotoxicity. It has also been reported that this level of mild heating cannot delay tumor growth or cause direct thermal radiosensitization (17). In a previous study, MTH mainly oxygenated the chronically hypoxic fraction, though it had less of an impact than carbogen inhalation (15). Moreover, it has been shown that cells intermediate in oxygenation are able to influence tumor response to radiation, and that such cells and hypoxic cell cytotoxins potentially constitute a significant proportion of solid tumors (21). Thus, it has been suggested that MTH changes certain chronically hypoxic fractions to a level intermediate between fully oxygenated and hypoxic through an increase in tumor blood flow, and at the same time distributes higher doses of TPZ and kills cells at these intermediate oxygen tensions (21). Indeed, MTH has been demonstrated to induce an increase in tumor $\mathrm{pO}_{2}$ (4), particularly in the oxygenation of Q cell populations (15), resulting from an improvement in the supply of oxygen via an increase in tumor blood flow. MTH has also been shown to sensitize total cells and, in particular, Q cells to the toxicity of TPZ when combined with or without $\gamma$-ray irradiation and/ or cisplatin (15). The present study also demonstrated that the combination with MTH is useful for enhancing TPZ toxicity in total cells and, in particular, Q cells, when combined with or without $\gamma$-ray irradiation or cisplatin treatment.

The Warburg effect refers to the observation that most cancer cells predominantly produce energy by glycolysis followed by lactic acid fermentation in the cytosol, rather than by oxidation of pyruvate in mitochondria like most normal cells (19). At present, the Warburg effect is thought to be i) the result of damage to the mitochondria in cancer, ii) an adaptation to low-oxygen environments within tumors, or iii) the result of cancer genes shutting down the mitochondria because they are involved in a cell apoptosis program that would otherwise kill cancerous cells (22). Since the use of MTH can improve intratumor oxygen supply via an increase in tumor blood flow, the Warburg effect is suppressed with MTH treatment. Thereby, the production of formaldehyde from HMTA under acidic conditions is also suppressed, resulting in a decrease in the amount of DNA-protein crosslink as a major form of DNA damage through exposure to formaldehyde. Thus, whether combined with or without $\gamma$-ray irradiation or cisplatin treatment, sensitivity to HMTA was reduced in combination with MTH in total and Q cells. This was particularly the case in $\mathrm{Q}$ cells, due to greater improvements in the oxygen supply through MTH in the Q compared to total cell population (15). Furthermore, whether $\gamma$-ray irradiation was administered at a dose of 4 or $20 \mathrm{~Gy}$, further combination with MTH on the combined effect of radiation with HMTA or TPZ had nearly the same effect. This means that the effect of combination with MTH is almost interchangeable, irrespective of radiation fraction size in fractionated radiotherapy. The findings observed in this study can be applied to fractionated radiotherapy with small fraction size, such as practically performed radiotherapy. 
It has previously been shown that total- and Q cell populations in SCC VII tumors are rich in acutely and chronically hypoxic fractions, respectively (15). Therefore, continuously administered HMTA or TPZ was more cytotoxic to tumor cells in vivo than single intraperitoneal administration, since the sensitizing effect on the acutely hypoxia-rich total cell population was added to the effect had on the chronically hypoxia-rich Q cell population (9). This is why the enhancement observed with continuous administration compared to single administration was more marked in the total cell population than in Q cells.

The presence of $\mathrm{Q}$ cells is thought to be due, in part, to hypoxia and the depletion of nutrition in the tumor core. This is another consequence of poor vascular supply (23), and might promote the formation of micronuclei in Q tumor cells, even without any treatment. Essentially, Q cells showed less sensitivity to $\gamma$-ray irradiation and cisplatin treatment (15), which means more $\mathrm{Q}$ cells than $\mathrm{P}$ cells survive after conventional radiotherapy or chemotherapy. Thus, the control of $\mathrm{Q}$ cells has a great impact on the outcome of anticancer treatment. As a result, conventional radiotherapy or chemotherapy in combination with HMTA or TPZ, which in and of themselves have significantly more toxicity to Q cells than to the total cell population, is thought to be useful. Taking into account that HMTA has a history of clinical use as an antiseptic for urinary tract infections $(13,14)$, it may have more potential than TPZ to be employed with conventional anticancer therapy. However, studies on the toxicity of HMTA in normal tissue must still be carried out for safety assurance. In terms of the tumor cell-killing effect as a whole, including intratumor $\mathrm{Q}$ cell control, continuously administered HMTA in combination with conventional radiotherapy and chemotherapy may be a promising treatment modality for refractory tumors due to its advantageous cytotoxic effects. However, HMTA combined with MTH should not be employed, due to a reduction in the radiosensitizing or chemotherapy sensitivity-enhancing effect observed when HMTA is used as a combined agent in conventional radiotherapy and chemotherapy.

\section{Acknowledgements}

This study was supported, in part, by a grant-in-aid for Scientific Research (C) (20591493) from the Japan Society for the Promotion of Science.

\section{References}

1. Hall EJ and Roizin-Towle L: Biological effects of heat. Cancer Res 44: 4708-4713, 1984.

2. Sapareto SA and Dewey WC: Thermal dose determination in cancer therapy. Int J Radiat Oncol Biol Phys 10: 787-800, 1984.
3. Oleson JR, Samulski TV, Leopold KA, et al: Sensitivity of hyperthermia trial outcomes to temperature and time: Implications for thermal goals of treatment. Int J Radiat Oncol Biol Phys 25: 289-297, 1993.

4. Song CW, Park HJ, Lee CK and Griffin R: Implications of increased tumour blood flow and oxygenation caused by mild temperature hyperthermia in tumor treatment. Int J Hyperthermia 21: 761-767, 2005.

5. Armour EP and Raaphorst GP: Long duration mild temperature hyperthermia and brachytherapy. Int J Hyperthermia 20: 175-189, 2004.

6. Kong G, Braun RD and Dewhirst MW: Hyperthermia enables tumor-specific nanoparticle delivery: effect of particle size. Cancer Res 60: 4440-4445, 2000.

7. Li CY and Dewhirst MW: Hyperthermia-regulated immunogene therapy. Int J Hyperthermia 18: 586-596, 2002.

8. Kinuya S, Yokoyama K, Michigishi T and Tonami N: Optimization of radioimmunotherapy interactions with hyperthermia. Int $\mathrm{J}$ Hyperthermia 20: 190-200, 2004.

9. Masunaga S, Nagasawa H, Uto Y, et al: The usefulness of continuous administration of hypoxic cytotoxin combined with mild temperature hyperthermia, with reference to effects on quiescent tumour cell populations. Int J Hyperthermia 21: 305-318, 2005.

10. Brown JM: Evidence of acutely hypoxic cells in mouse tumours and a possible mechanism of reoxygenation. Br J Radiol 52: 650-656, 1979.

11. McKeown SR, Cowen RL and Williams KJ: Bioreductive drugs: from concept to clinic. Clin Oncol 19: 427-442, 2007.

12. Adam M, Bayer C, Henke J, Grosu A, Molls M and Nieder C: Tirapazamine plus cisplatin and irradiation in a mouse model: improved tumor control at the cost of increased toxicity. J Cancer Res Clin Oncol 134: 137-146, 2008.

13. Ridpath JR, Nakamura J, Tano K, et al: Cells deficient in the Fanc/Brac pathway are hypersensitive to plasma levels of formaldehyde. Cancer Res 67: 11117-11122, 2007.

14. Swift LP, Cutts SM, Rephaeli A, Nudelman A and Phillips DR: Activation of adriamycin by the $\mathrm{pH}$-dependent formaldehydereleasing prodrug hexamethylenetetramine. MolCancerTherapeut 2: 189-198, 2003.

15. Masunaga S and Ono K: Significance of the response of quiescent cell populations within solid tumors in cancer therapy. J Radiat Res 43: 11-25, 2002.

16. Masunaga S, Tano K, Watanabe M, et al: Evaluation of the potential of hexamethylenetetramine, compared with tirapazamine, as a combined agent with $\gamma$-irradiation and cisplatin treatment in vivo. Br J Radiol 82: 392-400, 2009.

17. Nishimura Y, Ono K, Hiraoka M, et al: Treatment of murine SCC VII tumors with localized hyperthermia and temperature-sensitive liposomes containing cisplatin. Radiat Res 122: 161-167, 1990.

18. Hubal EA, Schlosser PM, Conolly RB and Kimbell JS: Comparison of inhaled formaldehyde dosimetry predictions with DNA-protein cross-link measurements in the rat nasal passages. Toxicol Appl Pharmacol 143: 47-55, 1997.

19. Kim JW and Dang CV: Cancer's molecular sweet tooth and the Warburg effect. Cancer Res 66: 8927-8930, 2006.

20. Iskandarova GT: Hygienic rationale for maximum permissible concentration of hexamethylenetetramine salt of 2-chloroethylphosphonic acid. Gig Sanit 10: 14-17, 1993.

21. Wouters BG and Brown JM: Cells at intermediate oxygen levels can be more important than the 'hypoxic fraction' in determining tumor response to fractionated radiotherapy. Radiat Res 147: 541-550, 1997,

22. Bertram JS: The molecular biology of cancer. Mol Aspects Med 21: 167-223, 2000.

23. Vaupel P: Tumor microenvironmental physiology and its implications for radiation oncology. Semin Radiat Oncol 14: 197-275, 2004. 